\title{
Quantitative Analyse von Korallengemeinschaften des Sanganeb-Atolls (mittleres Rotes Meer). II. Vergleich mit einem Riffareal bei Aqaba (nördliches Rotes Meer) am Nordrande des indopazifischen Riffgürtels
}

\author{
H. Schuhmacher ${ }^{1} \&$ H. Mergner ${ }^{2}$ \\ ${ }^{1}$ Universität GHS Essen, Lehrstuhl für Hydrobiologie; \\ Postfach 1037 64, D-4300 Essen, Bundesrepublik Deutschland \\ ${ }^{2}$ Ruhr-Universität Bochum, Lehrstuhl für Spezielle Zoologie; \\ Postfach 1021 48, D-4630 Bochum, Bundesrepublik Deutschland
}

\begin{abstract}
Quantitative analysis of coral communities of Sanganeb-Atoll (central Red Sea). II. Comparison with a reef area near Aqaba (northern Red Sea) at the northern margin of the Indopacific reef-belt. Quantitative studies of coral communities in the central and northern Red Sea were designed for comparison of the community structure in both areas. The central Red Sea provides reef-building Scleractinia and reef-inhabiting Alcyonaria with optimal temperature conditions, whereas the north tip of the Gulf of Aqaba $\left(29^{\circ} 30^{\prime} \mathrm{N}\right)$ represents the northernmost outpost of coral reefs in the Indian Ocean. It is generally assumed that coral diversity decreases towards the margins of the global reef-belt. In the Red Sea, generic diversity of hermatypic Scleractinia slightly decreases from the central to the northern part (51:48 genera); but cnidarian species abundance (species number per $25 \mathrm{~m}^{2}$ area) was found to increase from 62 to 98 species and the ShannonWiener diversity index increased from 2.58 to 3.67 with regard to colony number. The mean colony size was $189 \mathrm{~cm}^{2}$ at Sanganeb-Atoll, but only $52 \mathrm{~cm}^{2}$ at Aqaba. The mean numbers of colonies were inversely related: 662 per $25 \mathrm{~m}^{2}$ at Sanganeb-Atoll and 2028 at Aqaba. Uninhabited parts of the studied areas amounted to $47 \%$ at Sanganeb-Atoll and to $56 \%$ at Aqaba. The community structure of the studied areas indicates that occasional perturbations prevent the progress of the community towards a low-diversity equilibrium state. Since severe hydrodynamic damage is extremely rare in $10 \mathrm{~m}$ depth, major disturbances may occur by sedimentation, by the interference of grazers (e. $\mathrm{g}$. Diadema setosum) and due to overgrowth by space-competitors (mainly soft corals). These events are to be regarded as throwbacks in the process of monopolization of the area by well adapted species. Recovery from such perturbations (i.e. recolonization of dead areas) obviously takes place at different velocities in the northern and central Red Sea, for the mean water temperature at Aqaba is $5^{\circ} \mathrm{C}$ lower than in the central Red Sea. Hence the process of taking over a given space by a few species proceeds further in the central Red Sea than at its northern end. The increase in diversity per area towards high latitudes is comparable to that with depth. It is concluded from the great number of species at Aqaba that these reefs mark the northernmost outpost of the Indian Ocean only geographically but not ecophysiologically; they would occur at even higher latitudes, if the Gulf of Aqaba extended farther north.
\end{abstract}




\section{EINLEITUNG}

Für Korallenriffe gilt, daß Artenzahl und Diversität von hermatypischen Korallen zum Rand des Riffgürtels hin abnehmen (z. B. Stehli \& Wells, 1971; Rosen, 1971; Glynn, 1973). Für eine Überprüfung dieser Aussage bietet sich das Rote Meer geradezu an, denn es erstreckt sich als über $2270 \mathrm{~km}$ langer Graben vom tropischen Bereich weit nach Norden und beinhaltet mit den Riffen bei Aqaba und Eilat $\left(29^{\circ} 30^{\prime} \mathrm{N}\right)$ die nördlichsten Riffaußenposten des Indischen Ozeans.

Publikationen aus jüngerer Zeit (Loya \& Slobodkin, 1971; Loya, 1972; Mergner \& Schuhmacher, 1974, 1981) haben für das Nordende des Golfes von Aqaba eine bemerkenswert hohe Artenfülle und Diversität von Korallen nachgewiesen, die vorübergehend sogar die des mittleren Roten Meeres übertrafen. Da sich aber im Umfang von regionalen Artenlisten auch die Intensität der Bearbeitung eines Raumes widerspiegelt und die Bezugsfläche der erfaßten Artendiversität nicht angegeben wird, sagen solche Listen (Rosen, 1971; Scheer, 1971; Scheer \& Pillai, 1983; Scheer, 1984) über die Diversität der Besiedlung einzelner Riffabschnitte wenig aus. Es stellte sich daher die Aufgabe, Artenzusammensetzung und Struktur der Korallenbesiedlung methodisch in identischer Weise an ausgewählten Untersuchungsflächen am Nordende und im mittleren Roten Meer vergleichend zu analysieren.

Artenzahl und -diversität, Koloniezahl und -größe der sessilen Cnidaria (der mit Abstand bedeutendsten Organismengruppe im Riff) wurden in einheitlich umschriebenen Flächen bei Aqaba (Mergner \& Schuhmacher, 1981) und am Sanganeb-Atoll vor der sudanesischen Küste (Mergner \& Schuhmacher, 1985) detailliert erfaßt.

Der Wissensstand über die Korallenriffe im nördlichen Golf von Aqaba und im mittleren Roten Meer entwickelte sich in unterschiedlicher Weise. Das mittlere Rote Meer war schon früh Ziel entsprechender Untersuchungen: Von Europa aus relativ leicht zugänglich, wurden die Riffe an der ägyptischen und sudanesischen Küste bereits von Forskål (1775), Ehrenberg (1834a, b), Klunzinger (1872, 1877, 1879a, b) und Crossland $(1907,1935,1938)$ untersucht. Crossland (1939) bezweifelte hingegen generell die Existenz von Riffen im Golf von Aqaba. Erst seit dem Jahre 1970 wurden die Riffe und ihre Korallenbesiedlung im Umkreis der meeresbiologischen Institute von Eilat und Aqaba bekannt und intensiv bearbeitet. Eine Ubersicht der korallen- und riffökologischen Publikationen geben Scheer \& Pillai (1983), Mergner (1984) und Scheer (1984). Bei frühen biogeographischen Vergleichen, so auch zu den Teilbereichen des Roten Meeres, (Stehli \& Wells, 1971; Rosen, 1971; Scheer, 1971) lagen hauptsächlich Korallenaufsammlungen zugrunde, die beim Waten über das Riffdach oder vom Boot aus gewonnen worden waren. Zu diesem Zeitpunkt war der Korallenbestand des Golfes von Aqaba noch nahezu unbekannt. Dieser Wissensrückstand wurde durch intensive Tauchuntersuchungen nicht nur aufgeholt; die Artenvielfalt am Nordende des Roten Meeres erschien zeitweise sogar größer als die im mittleren Roten Meer. Erst entsprechend intensive Tauchaufsammlungen vor der sudanesischen Küste (Head, 1980; Vine \& Vine, 1980; Kühlmann, 1983; Mergner \& Schuhmacher, 1985) brachten das bekannte Arteninventar näher an den (noch verborgenen) tatsächlichen Artenbestand heran. Dem aktuellen Bearbeitungsstand gemäß schwankt auch die Rangfolge der Arten- bzw. Gattungsvielfalt: Scheer \& Pillai (1983) nennen für den Golf von Aqaba 48 Scleractinia-Gattungen mit 127 Arten, für das mittlere Rote Meer jedoch nur 40 Gattungen mit 116 Arten. 
Für letztgenannten Bereich erhöht sich dann bei Scheer (1984) die Zahl auf 51 hermatypische Gattungen.

Die im Verlauf der Zeit wechselnde Kenntnis des relativen Artenreichtums im mittleren und nördlichen Roten Meer wurde deshalb mit einigen Einzelheiten ausgeführt, um die eingeschränkte Aussagekraft eines bloßen Vergleichs von Artenlisten darzulegen. Um eine regional unterschiedliche Diversität in der Korallenbesiedlung tatsächlich zu erfassen, wurde vorliegende Untersuchung durchgeführt.

Die Besiedlung von $5 \times 5 \mathrm{~m}$ großen Ausschnitten von Korallenbeständen wurde in Riffen bei Aqaba und am Sanganeb-Atoll quantitativ analysiert. Um störende Einflüsse durch unterschiedliches Lichtangebot möglichst zu vermeiden, wurden alle Untersuchungsareale (im Folgenden auch Testquadrate, TQs oder Testareale genannt) in annähernd gleicher Tiefe um $10 \mathrm{~m}$ angelegt. Bei dem im Roten Meer gegebenen klaren Wasser ist in dieser Tiefe der Korallenwuchs oft am intensivsten (Barnes \& Taylor, 1973; Mergner \& Svoboda, 1977; Svoboda, 1978), da hier weder durch zu geringe noch zu intensive Beleuchtung Lichthemmung eintritt. Außerdem sind in $10 \mathrm{~m}$ Tiefe die Tauchzeiten noch nicht nennenswert eingeschränkt. Hingegen differierten die Testquadrate hinsichtlich der vorherrschenden Wasserbewegung und der Wassertemperatur. Die Frage war, ob sich insbesondere der großräumig wirkende abiotische Faktor Temperatur (vgl. Abb. 1), d.h. der Temperaturabfall vom mittleren zum nördlichen Roten Meer, in einer entsprechenden Veränderung der Artenzahl und Diversität der Riffbesiedlung niederschlagen würde.

\section{UNTERSUCHUNGSGEBIETE UND METHODIK}

Eine ausführliche Beschreibung der Naturverhältnisse und Korallenriffe von Aqaba gaben Mergner \& Schuhmacher (1974). Von denselben Autoren wurde 1981 die quantitative Analyse der Besiedlung eines $25 \mathrm{~m}^{2}$ Ausschnittes (Testquadrat U-7 genannt) aus dem Vorriff bei $10 \mathrm{~m}$ Tiefe vorgelegt. Es gilt im vorliegenden Vergleich als Referenzareal für das Nordende des Roten Meeres. Die Riffe bei Aqaba sind - verglichen mit denen im südlichen Golf von Aqaba und erst recht im mittleren Roten Meer - schwach ausgebildet. Das Saumriff in der Nähe von Testquadrat U-7 sitzt pultförmig dem ufernahen, nur drei bis fünf Meter tiefen Meeresboden auf (Abb. 2). Der seewärts zunächst mäßig abfallende Meeresboden ist von niedrigem Korallenbewuchs mit eingesprengten Sandinseln bestanden. Eine Tiefe von $10 \mathrm{~m}$ für das Untersuchungsareal wird erst ca. $70 \mathrm{~m}$ vor dem Riffhang erreicht. Dieser Bereich zählt zum mittleren Vorriff (vgl. Mergner \& Schuhmacher, 1974). Die Wassertemperatur schwankt zwischen $21^{\circ} \mathrm{C}$ im Winter und $25^{\circ}-26^{\circ} \mathrm{C}$ im Sommer. Die Windwellen aus $\mathrm{N}-\mathrm{NNW}$ haben bis zur Riffkante eine Anlaufstrecke von maximal $10 \mathrm{~km}$, sind gewöhnlich 0,3-0,5 $\mathrm{m}$ hoch und werden mit einer Windstärke von durchschnittlich 7,2 Kn (3,2 Bft) angetrieben. Im Vorriff herrscht eine Rifflängsströmung $\left(3-10 \mathrm{~cm} \cdot \mathrm{s}^{-1}\right)$, die entweder der Oberflächenströmung entgegen verläuft (Mergner \& Schuhmacher, 1974) oder mit ihr nach Süden setzt (Hulings, 1979).

Die zum Vergleich herangezogenen Referenzareale aus dem mittleren Roten Meer sind die vier Testquadrate am Sanganeb-Atoll, die von Mergner \& Schuhmacher (1985) ausführlich dargestellt wurden. Das Sanganeb-Atoll liegt ca. $28 \mathrm{~km}$ nordöstlich von Port Sudan vor der sudanesischen Küste vermutlich auf einem Horst, der beim Einbruch des 


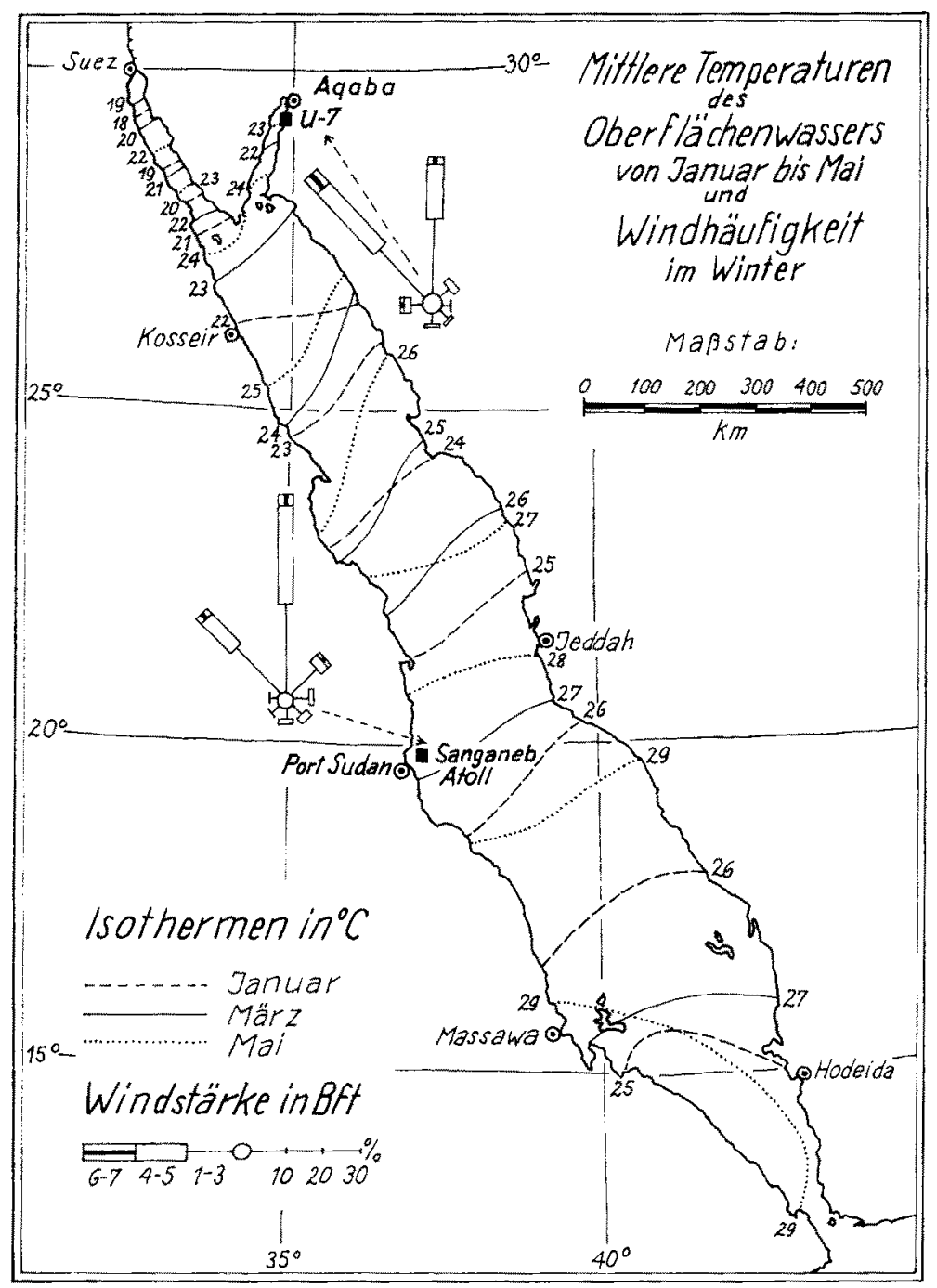

Abb. 1. Karte des Roten Meeres mit den Monatsisothermen des Oberflächenwassers für Januar, März und Mai sowie mit der Windhäufigkeit (Windrichtung und Windstärke in Bft) im Nordwinter

Rotmeergrabens emporgehoben wurde. Wie Abbildung 3 zeigt, steigt das SanganebAtoll steil aus $800-650 \mathrm{~m}$ Wassertiefe auf. Der in der Nordsüdrichtung stark verlängerte Riffring erreicht auf der relativ windgeschützten Westseite nicht überall den Wasserspiegel, sondern läßt eine bis $14 \mathrm{~m}$ tiefe Passage frei. Die brandungsexponierte Nordund Ostseite hingegen ist ringsum durch einen breiten, proliferierenden Riffring gekennzeichnet. Die Entstehung einer südlichen, von der Hauptlagune abgetrennten flachen Wanne und der breiten südlichen Riffplattform geht möglicherweise auf Erosion während eiszeitlichen Tiefstandes des Meeresspiegels zurück.

Der Riffhang fällt an der NO-Außenseite fast senkrecht auf mehr als $50 \mathrm{~m}$ Tiefe ab 


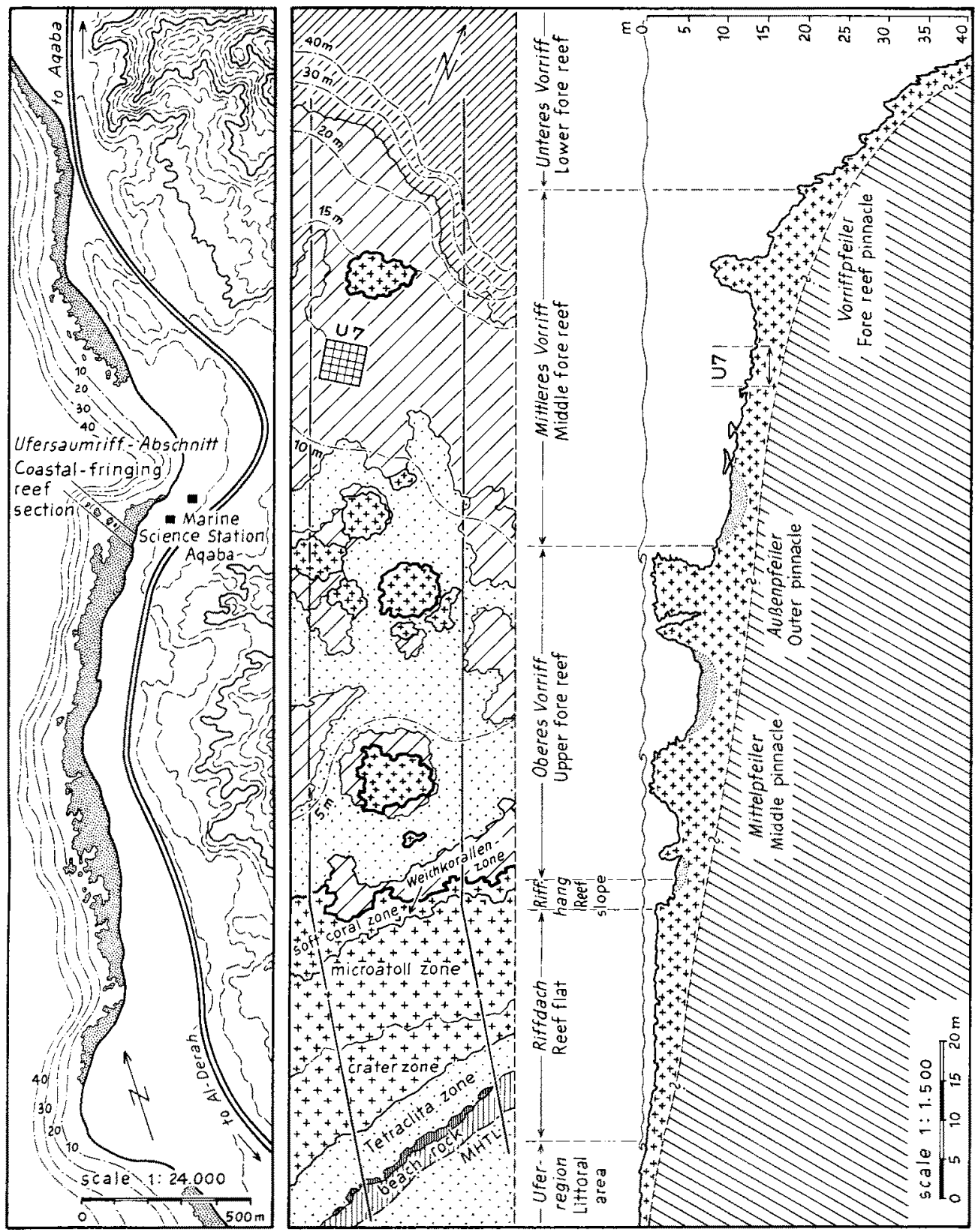

Abb. 2. Riffregion bei Aqaba mit Lage des Testareals U-7 im Ufersaumriff unmittelbar südlich der Marine Science Station (links), in der Aufsicht (Mitte) und im Profil (rechts). (Nach Mergner \& Schuhmacher, 1981) 
und an der S-Außenseite auf $40 \mathrm{~m}$, der Innenhang des Riffringes dagegen nur auf 10-15 $m$ zum Lagunenboden, der sich erst allmählich bis auf $48 \mathrm{~m}$ vertieft. Die in derselben Tiefenstufe von etwa $10 \mathrm{~m}$ eingerichteten Testquadrate befinden sich also in verschiedenen Riffabschnitten: im mittleren Vorriff bei Aqaba, in der Mitte des Riffhanges an den Sanganeb-Außenriffen und dicht über dem Lagunenboden an den Innenriffen. Die Wassertemperatur am Sanganeb-Atoll schwankt zwischen $25^{\circ} \mathrm{C}$ im Winter und $29^{\circ}-30^{\circ} \mathrm{C}$ im Sommer.

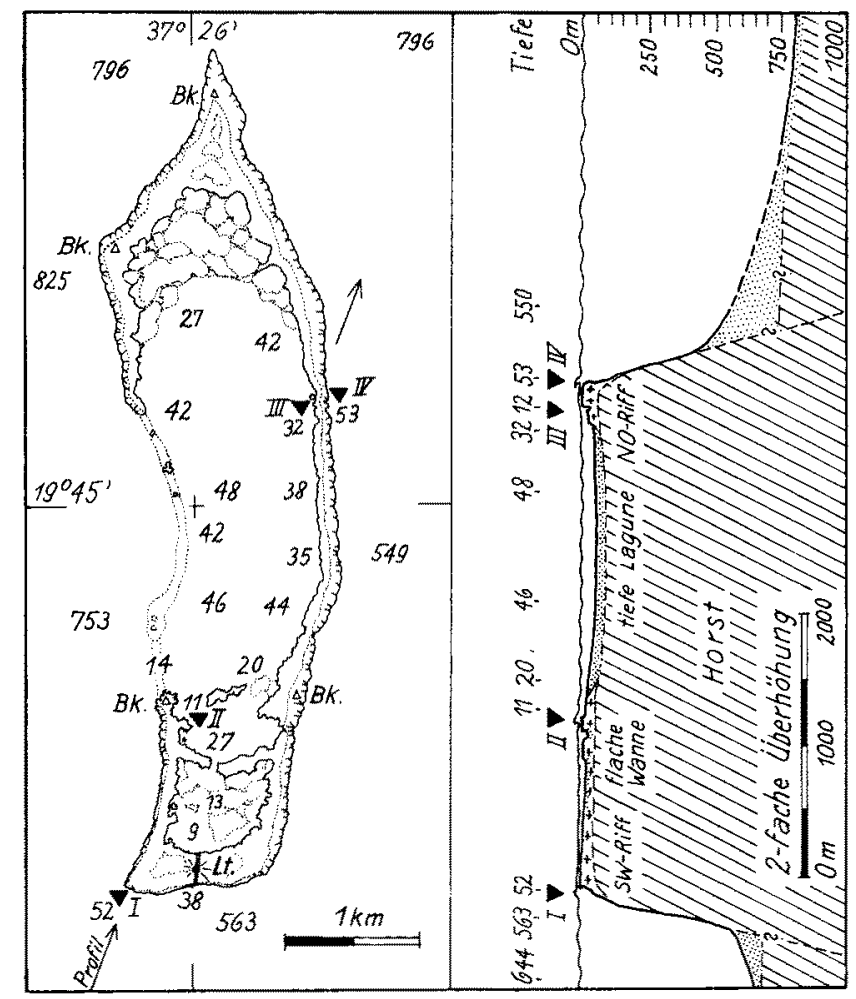

Abb. 3. Karte (links) und Profil (rechts) des Sanganeb-Atolls bei Port Sudan mit Lage der Testareale I-IV. (Karte nach Luftbild: Abb. 2 aus Mergner \& Schuhmacher, 1985)

Das Sanganeb-Atoll wird ganzjährig von überwiegend nördlichen Winden getroffen und nur gelegentlich aus südlicher bis südöstlicher Richtung. Die mit einer Anlaufstrecke von mehr als $1000 \mathrm{~km}$ auf das Atoll treffenden Windwellen und Dünung werden durch täglich regelmäßig wechselnde Land-Seebrisen (im Winter aus NW bzw. NO) nur vorübergehend und mit unterschiedlicher Intensität in ostwestlicher Richtung abgelenkt. Häufig herrscht im Sommer in dieser Region nahezu völlige Windstille oder nur eine leichte Brise. Demzufolge wird der NO-Rand des Atolls, vor allem im Winter, fast ständig von mehr als $1 \mathrm{~m}$ hohen Wellen getroffen, die geschützte Südseite dagegen nur gelegentlich von meist nur 0,5 m hohen Wellen. Entsprechend der unterschiedlichen Brandungsstärke reichen Brandungsrücklauf und Oszillationsbewegungen am NO- 
Außenhang bis in $10 \mathrm{~m}$ Tiefe; am Südhang überwiegt hingegen in dieser Tiefe eine Rifflängsströmung.

Eine ausführliche Darstellung, wie die untersuchten Besiedlungsgemeinschaften in beiden Regionen erfaßt wurden, findet sich bei Mergner \& Schuhmacher $(1981,1985)$, so daß hier nur die wesentlichsten Punkte wiederholt werden müssen. Ein $5 \times 5 \mathrm{~m}$ großes Untersuchungsareal wurde durch Nylonleinen abgegrenzt und in 25 Einzelquadrate unterteilt. Jedes Einzelquadrat wurde zentrisch photographiert und mit Hilfe wasserfester Papierphotoabzüge unter Wasser genau kartiert. Die Identität der einzelnen Kolonien wurde sofort - in situ - oder nach Entnahme von Proben festgestellt. Für den Vergleich der Flächenbedeckung der einzelnen Untersuchungsareale ist es ohne Belang, daß durch diese Methode nicht die tatsächliche dreidimensionale Oberfläche, sondern ihre Projektion in die Ebene erfaßt wird.

Die Untersuchungen bei Aqaba wurden im Februar 1976 (mit Ergänzungen bis zum Herbst 1977), diejenigen am Sanganeb-Atoll im Februar und März 1980 durchgeführt.

\section{ERGEBNISSE}

In den Testarealen I-IV am Sanganeb und U-7 bei Aqaba wurden die in Tabelle 1 aufgeführten Cnidaria-Arten gefunden.

Im Vergleich zur Artenliste aus dem U-7 von Mergner \& Schuhmacher (1981) ergaben sich einige Änderungen in der systematischen Zuordnung: Seriatopora angulata und Seriatopora sp. werden zu $S$, hystrix gestellt. Pocillopora danae ist synonym zu $P$. damicornis, Psammocora superficialis $=P$. profundacella, Acropora clavigera $=A$. granulosa, $A$. scandens $=A$. pharaonis, Montipora danae $=M$. meandrina, $M$. eilatensis $=M$. stilosa var. eilatensis, Pavona danai $=P$. cactus, Gardineroseris ponderosa $=G$. planulata, Platygyra lamellina $=P$. daedalea, Lobophyllia costata $=L$. hemprichii und Mycedium tubifex $=M$. elephantotus. Echinopora gemmacea fruticulosa wird als Varietät neben der Nominatform stets besonders ausgewiesen, da es im Gegensatz zu dieser eine verzweigte Wuchsform besitzt. Ferner ist die Identität von Montastrea forskaelana zumindest zweifelhaft; dieselbe Probe (aus U-7) wird von Wijsman-Best (1977, 1980) unterschiedlich beurteilt; Scheer \& Pillai (1983) halten M. forskaelana für eine Wuchsform von Echinopora gemmacea.

Die Artenliste in Tabelle 1 schließt auch die Alcyonaria mit ein, obwohl in konventionellen Korallenlisten (u. a. Wells, 1954; Rosen, 1971; Scheer, 1971) nur die Scleractinia, aber auch die Hydrocorallia und die Orgelkoralle Tubipora musica aufgeführt werden. Die allgemeine Gewohnheit, nur Hartskelett bildende Cnidaria zu berücksichtigen, erfaßt jedoch nur die dem Riffaufbau förderlichen Arten. Wir berücksichtigen hier auch die Weichkorallen, da sie nicht nur beim Zerfall reichlich Skleritmaterial als Sediment liefern, sondern vor allem einen mengenmäßig gewichtigen Anteil an der Flächenbedeckung haben (gelegentlich mehr als $50 \%$ der durch Cnidaria besiedelten Riffoberfläche) und als Raumkonkurrenten der Hartskelettbildner gerade den weiteren Kalkzuwachs erheblich behindern können. Der tatsächliche Riffzuwachs ist nämlich stets die Resultierende aus dem Wechselspiel von kalkaufbauenden und den Aufbau behindernden bzw. abtragenden Kräften. Außer den Cnidaria fanden sich als Besiedler der Testareale noch Kalkalgen, Porifera, Bivalvia, Bryozoa und Ascidia, wenn auch in 
Tab. 1. Cnidaria-Besiedlung der vier Testareale des Sanganeb-Atolls und des U-7 bei Aqaba

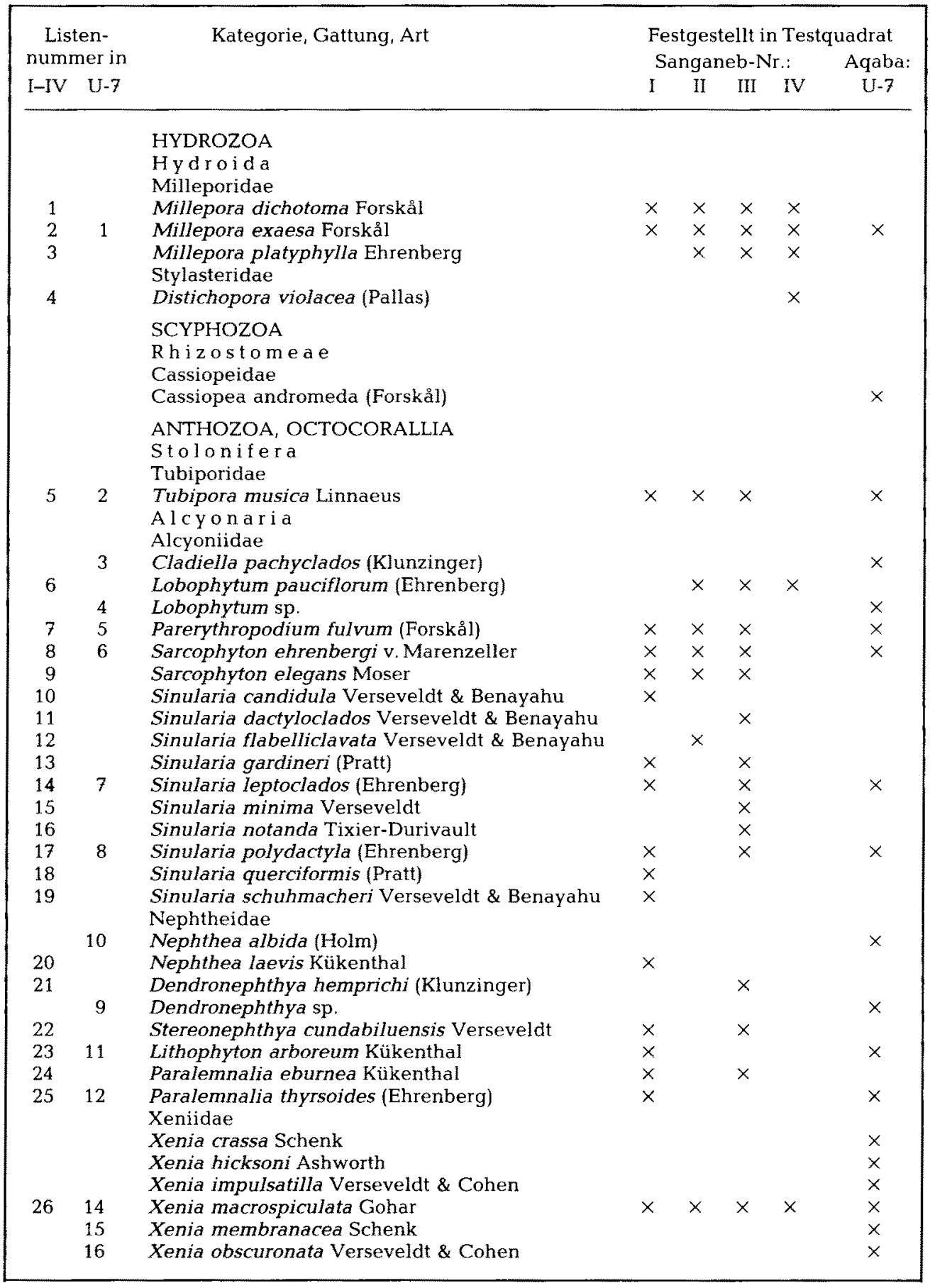


Tab. 1 (Fortsetzung)

\begin{tabular}{|c|c|c|c|c|c|c|c|}
\hline \multirow{2}{*}{\multicolumn{2}{|c|}{$\begin{array}{l}\text { Listen- } \\
\text { nummer in }\end{array}$}} & \multirow[t]{3}{*}{ Kategorie, Gattung, Art } & \multicolumn{5}{|c|}{ Festgestellt in Testquadrat } \\
\hline & & & \multicolumn{4}{|c|}{ Sanganeb-Nr.: } & \multirow{2}{*}{$\begin{array}{c}\text { Aqaba: } \\
\text { U-7 }\end{array}$} \\
\hline $\mathrm{I}-\mathrm{IV}$ & U-7 & & I & II & III & IV & \\
\hline \multirow[t]{2}{*}{27} & 18 & Xenia umbellata Lamarck & $x$ & & & & $x$ \\
\hline & 17 & Xenia spec. & & & & & $\times$ \\
\hline 28 & 13 & Heteroxenia fuscescens (Ehrenberg) & $x$ & & & & $x$ \\
\hline 29 & & Anthelia fishelsoni Verseveldt & $x$ & $x$ & & & \\
\hline 30 & & Anthelia glauca Lamarck & $x$ & & $x$ & & \\
\hline \multirow[t]{2}{*}{31} & & Sympodium caeruleum Ehrenberg & $\times$ & & & $\times$ & \\
\hline & & $\begin{array}{l}\text { Gorgon aria } \\
\text { Gorgoniidae }\end{array}$ & & & & & \\
\hline \multirow[t]{9}{*}{32} & & Clathraria rubrinodis Gray & $x$ & & & $\times$ & \\
\hline & & ANTHOZOA, HEXACORALLIA & & & & & \\
\hline & & Actiniaria & & & & & \\
\hline & & Actiniidae & & & & & \\
\hline & & Gyrostoma helianthus (Ehrenberg) & & & & & $x$ \\
\hline & & Gyrostoma quadricolor (Leuckart) & & & & & $x$ \\
\hline & & Radianthus koseirensis (Klunzinger) & & & & & $x$ \\
\hline & & Scleractinia & & & & & \\
\hline & & Thamnasteriidae & & & & & \\
\hline 33 & & Psammocora haimeana Milne Edwards \& Haime & $x$ & $\times$ & $x$ & $x$ & \\
\hline \multirow[t]{2}{*}{34} & & Psammocora nierstraszi v. d. Horst* & $\times$ & & & $x$ & \\
\hline & 19 & $\begin{array}{l}\text { Psammocora profundacella Gardiner } \\
\text { Astrocoeniidae }\end{array}$ & & & & & $x$ \\
\hline \multirow[t]{2}{*}{35} & & Stylocoeniella armata (Ehrenberg) ${ }^{*}$ & & & & $\times$ & \\
\hline & & Pocilloporidae & & & & & \\
\hline 36 & 25 & Stylophora pistillata (Esper) & & $x$ & $\times$ & $x$ & $\times$ \\
\hline 37 & 23 & Seriatopora caliendrum Ehrenberg ${ }^{\circ}$ & $\times$ & $x$ & $x$ & $x$ & $x$ \\
\hline 38 & 22,24 & Seriatopora hystrix Dana & $x$ & $\times$ & & & $x$ \\
\hline 39 & 20,21 & Pocillopora damicornis (Linnaeus) & $x$ & & $\times$ & & $x$ \\
\hline 40 & & $\begin{array}{l}\text { Pocillopora verrucosa (Ellis \& Solander) } \\
\text { Acroporidae }\end{array}$ & & $x$ & $x$ & $x$ & \\
\hline 41 & 35 & Astraeopora myriophthalma (Lamarck) & & & $x$ & & $x$ \\
\hline 42 & & Acropora capillaris (Klunzinger) & & $\times$ & & & \\
\hline \multirow[t]{4}{*}{43} & & Acropora corymbosa (Lamarck) & & & $x$ & $x$ & \\
\hline & 27 & Acropora eurystoma (Klunzinger) & & & & & $x$ \\
\hline & 28 & Acropora forskali (Ehrenberg) & & & & & $\times$ \\
\hline & 26 & Acropora granulosa (Milne Edwards \& Haime) & & & & & $\times$ \\
\hline 44 & & Acropora cf. haimei (Milne Edwards \& Haime) & & & & $x$ & \\
\hline 45 & 29 & Acropora hemprichi (Ehrenberg) & $\times$ & $x$ & $x$ & $x$ & $x$ \\
\hline 46 & 30 & Acropora humilis (Dana) & & $\times$ & $x$ & $x$ & $x$ \\
\hline 47 & & Acropora hyacinthus (Dana) & & $\times$ & $x$ & $x$ & $x$ \\
\hline 48 & 31 & Acropora pharaonis (Milne Edwards \& Haime) & $x$ & $\times$ & & $x$ & $x$ \\
\hline 49 & 32 & Acropora squarrosa (Ehrenberg)* & & & & $x$ & $x$ \\
\hline 50 & & Acropora superba (Klunzinger) & & & & $x$ & \\
\hline 51 & 33 & Acropora variabilis (Klunzinger) & $x$ & $x$ & $x$ & $x$ & $x$ \\
\hline 52 & 34 & Acropora sp. & & & $x$ & $x$ & $\times$ \\
\hline 53 & 37 & Montipora effusa (Dana) & $x$ & $x$ & & & $x$ \\
\hline 54 & & Montipora ehrenbergi Verrill & $x$ & $\times$ & $x$ & & \\
\hline 55 & & Montipora granulosa Bernard & & $\times$ & & & \\
\hline 56 & 36,39 & Montipora meandrina (Ehrenberg) & $x$ & $x$ & & & $x$ \\
\hline 57 & 40 & Montipora monasteriata (Forskål) & $x$ & $x$ & & $x$ & $x$ \\
\hline \multirow[t]{2}{*}{58} & 41 & Montipora stilosa (Ehrenberg) & $\times$ & $x$ & & & $x$ \\
\hline & 38 & Montipora stilosa var. eilatensis & & & & & $x$ \\
\hline
\end{tabular}


Tab. 1 (Fortsetzung)

\begin{tabular}{|c|c|c|c|c|c|c|c|}
\hline \multirow{2}{*}{\multicolumn{2}{|c|}{$\begin{array}{l}\text { Listen- } \\
\text { nummer in }\end{array}$}} & \multirow[t]{3}{*}{ Kategorie, Gattung, Art } & \multicolumn{5}{|c|}{ Festgestellt in Testquadrat } \\
\hline & & & \multicolumn{4}{|c|}{ Sanganeb-Nr.: } & \multirow{2}{*}{$\begin{array}{l}\text { Aqaba: } \\
\text { U-7 }\end{array}$} \\
\hline I-IV & $\mathrm{U}-7$ & & I & II & III & IV & \\
\hline 59 & & Montipora tuberculosa (Lamarck) & $x$ & & $x$ & $x$ & \\
\hline 60 & 42 & Montipora venosa (Ehrenberg) ${ }^{*}$ & $x$ & & $x$ & & $x$ \\
\hline 61 & & Montipora verrucosa (Lamarck)* & & $x$ & & & \\
\hline \multirow[t]{2}{*}{62} & 43 & $\begin{array}{l}\text { Montipora sp. } \\
\text { Aqariciidae }\end{array}$ & $x$ & $x$ & $\times$ & & $x$ \\
\hline & 47 & Pavona cactus (Forskål) & & & & & $x$ \\
\hline \multirow[t]{2}{*}{63} & & Pavona clavus (Dana) & & & & $x$ & \\
\hline & 48 & Pavona decussata (Dana) & & & & & $\times$ \\
\hline 64 & & Pavona divaricata Lamarck & & & & $\times$ & \\
\hline 65 & & Pavona explanulata (Lamarck) & & $\times$ & & & \\
\hline 66 & & Pavona maldivensis (Gardiner) & $x$ & & $x$ & $x$ & \\
\hline 67 & 49 & Pavona varians Verrill & $\times$ & & $x$ & $\times$ & $x$ \\
\hline \multirow[t]{2}{*}{68} & & Leptoseris mycetoseroides Wells & & $x$ & $x$ & & \\
\hline & 45 & Leptoseris $\mathrm{sp}$ & & & & & $x$ \\
\hline \multirow[t]{2}{*}{69} & 44 & Gardineroseris planulata (Dana) & $x$ & $x$ & $x$ & $x$ & $x$ \\
\hline & 46 & $\begin{array}{l}\text { Pachyseris speciosa (Dana) } \\
\text { Siderastreidae }\end{array}$ & & & & & $x$ \\
\hline \multirow[t]{4}{*}{70} & 50 & Coscinaraea monile (Forskål) & & $x$ & $x$ & & $\times$ \\
\hline & & Fungiidae & & & & & \\
\hline & 51 & Cycloseris doederleini (v. Marenzeller) & & & & & $x$ \\
\hline & 52 & Cycloseris sp. & & & & & $x$ \\
\hline 71 & & Fungia echinata (Pallas) & & $x$ & $\times$ & & \\
\hline \multirow[t]{2}{*}{72} & 53 & Fungia fungites (Linnaeus) & & $x$ & $x$ & $x$ & $\times$ \\
\hline & 54 & Fungia granulosa Klunzinger & & & & & $x$ \\
\hline 73 & & Fungia klunzingeri Doederlein & & & $\times$ & & \\
\hline 74 & & Fungia scutaria Lamarck & & & & $x$ & \\
\hline \multirow[t]{2}{*}{75} & & Herpolitha limax (Esper) & & $x$ & & & \\
\hline & & Poritidae & & & & & \\
\hline 76 & 55 & Alveopora daedalea (Forskål) & & & $x$ & & $x$ \\
\hline \multirow[t]{2}{*}{77} & & Goniopora minor Crossland* & $\times$ & & & $x$ & \\
\hline & 56 & Goniopora planulata (Ehrenberg) & & & & & $x$ \\
\hline \multirow{3}{*}{78} & & Goniopora tenuidens Quelch ** & $x$ & & & & \\
\hline & 57 & Goniopora sp. & & & & & $x$ \\
\hline & 58 & Porites (Synarea) convexa Verrill & & & & & $x$ \\
\hline 79 & & Porites echinulata Klunzinger & $\times$ & & $\times$ & & \\
\hline 80 & 59 & Porites lutea Milne Edwards \& Haime & $x$ & $x$ & $x$ & $x$ & $x$ \\
\hline 81 & & Porites solida (Forskål) & $x$ & $x$ & $x$ & $x$ & \\
\hline 82 & & Porites (Synarea) undulata Klunzinger & $x$ & $x$ & $x$ & & \\
\hline \multirow[t]{2}{*}{83} & 60 & $\begin{array}{l}\text { Porites sp. } \\
\text { Faviidae }\end{array}$ & $x$ & $x$ & $x$ & $x$ & $x$ \\
\hline & 61 & Caulastrea tumida Matthai & & & & & $x$ \\
\hline 84 & 69 & Favia amicorum (Milne Edwards \& Haime)* & $x$ & & & & $x$ \\
\hline 85 & 70 & Favia favus (Forskål) & & $x$ & & & $x$ \\
\hline 86 & 71 & Favia laxa (Klunzinger) & $x$ & $x$ & & & $x$ \\
\hline 87 & 72 & Favia pallida (Dana) & $\times$ & & $x$ & $x$ & $x$ \\
\hline 88 & & Favia rotumana (Gardiner) * * & & & & $x$ & \\
\hline 89 & & Favia speciosa (Dana) & & & $x$ & $x$ & \\
\hline \multirow[t]{3}{*}{90} & 73 & Favia stelligera (Dana) & & $x$ & & $x$ & $x$ \\
\hline & 74 & Favia sp. & & & & & $x$ \\
\hline & 75 & Favites abdita (Ellis \& Solander) & & & & & $x$ \\
\hline 91 & & Favites complanata (Ehrenberg) & $x$ & $x$ & $x$ & & \\
\hline
\end{tabular}


Tab. 1 (Fortsetzung)

\begin{tabular}{|c|c|c|c|c|c|c|c|}
\hline \multirow{2}{*}{\multicolumn{2}{|c|}{$\begin{array}{l}\text { Listen- } \\
\text { nummer in }\end{array}$}} & \multirow[t]{3}{*}{ Kategorie, Gattung, Art } & \multicolumn{5}{|c|}{ Festgestellt in Testquadrat } \\
\hline & & & \multicolumn{4}{|c|}{ Sanganeb-Nr.: } & \multirow{2}{*}{$\begin{array}{l}\text { Aqaba: } \\
\text { U-7 }\end{array}$} \\
\hline I-IV & $\mathrm{U}-7$ & & I & II & III & IV & \\
\hline 92 & & Favites flexuosa (Dana) & $\times$ & & $x$ & $x$ & \\
\hline 93 & & Favites halicora (Ehrenberg) & & & $x$ & & \\
\hline \multirow[t]{2}{*}{94} & & Favites pentagona (Esper) & $x$ & & $x$ & & \\
\hline & 76 & Favites peresi Faure \& Pichon & & & & & $\times$ \\
\hline \multirow[t]{2}{*}{95} & & Favites rotundata Veron, Pichon \& Wijsman-Best & $x$ & & & $\times$ & \\
\hline & 77 & Favites sp. & & & & & $x$ \\
\hline 96 & 78 & Goniastrea edwardsi Chevalier & & $x$ & & & $x$ \\
\hline 97 & 79 & Goniastrea pectinata (Ehrenberg) & $\times$ & $x$ & $x$ & $x$ & $\times$ \\
\hline \multirow[t]{2}{*}{98} & 80 & Goniastrea retiformis (Lamarck) & & $x$ & & $x$ & $x$ \\
\hline & 81 & Goniastrea sp. & & & & & $x$ \\
\hline 99 & 87 & Platygyra daedalea (Ellis \& Solander) & & $\times$ & & & $x$ \\
\hline 100 & & Leptoria phrygia (Ellis \& Solander) & & & & $x$ & \\
\hline \multirow{2}{*}{101} & & Oulophyllia crispa (Lamarck) & & $\times$ & & & \\
\hline & 82 & Hydnophora exesa (Pallas) & & & & & $x$ \\
\hline 102 & & Hydnophora microconus (Lamarck) & & $\times$ & & & \\
\hline 103 & 83 & Leptastrea bottae (Milne Edwards \& Haime) & $x$ & $\times$ & $x$ & & $x$ \\
\hline 104 & 84 & Leptastrea purpurea (Dana) & $\times$ & $x$ & & & $x$ \\
\hline 105 & 85 & Leptastrea transversa Klunzinger & & $\times$ & & & $x$ \\
\hline 106 & 62 & Cyphastrea chalcidicum (Forskål) & $\times$ & $\times$ & $\times$ & & $\times$ \\
\hline 107 & 63 & Cyphastrea microphthalma (Lamarck) & $x$ & $\times$ & & & $\times$ \\
\hline \multirow[t]{2}{*}{108} & 64 & Cyphastrea serailia (Forskål) & $x$ & $\times$ & & & $\times$ \\
\hline & 65 & Cyphastrea sp. & & & & & $x$ \\
\hline 109 & 66 & Echinopora gemmacea (Lamarck) & $x$ & $\times$ & $x$ & $x$ & $x$ \\
\hline 110 & 67 & Echinopora gemmacea var. fruticulosa & & $x$ & & & $\times$ \\
\hline \multirow[t]{2}{*}{111} & 68 & Echinopora lamellosa (Esper) & & $x$ & & & $x$ \\
\hline & 86 & $\begin{array}{l}\text { Montastrea forskaelana (Milne Edwards \& Haime) } \\
\text { Oculinidae }\end{array}$ & & & & & $\times$ \\
\hline 112 & & Galaxea astreata (Lamarck) & & $x$ & & & \\
\hline \multirow[t]{2}{*}{113} & 88 & $\begin{array}{l}\text { Galaxea fascicularis (Linnaeus) } \\
\text { Merulinidae }\end{array}$ & $\times$ & $x$ & $x$ & $\times$ & $x$ \\
\hline & 89 & $\begin{array}{l}\text { Merulina ampliata (Ellis \& Solander) } \\
\text { Mussidae }\end{array}$ & & & & & $\times$ \\
\hline 114 & & Scolymia vitiensis Brueggemann & $\times$ & & & & \\
\hline 115 & 91 & Lobophyllia corymbosa (Forskăl) & & $x$ & $x$ & & $x$ \\
\hline 116 & 92,93 & Lobophyllia hemprichil (Ehrenberg) & & $x$ & & & $x$ \\
\hline 117 & & Lobophyllia pachysepta Chevalier** & & $x$ & & & \\
\hline 118 & 90 & Acanthastrea echinata (Dana) & $x$ & & & $x$ & $x$ \\
\hline 119 & & $\begin{array}{l}\text { Symphyllia erythraea (Klunzinger) } \\
\text { Pectiniidae }\end{array}$ & $x$ & & $x$ & $x$ & \\
\hline 120 & 95 & Mycedium elephantotus (Pallas) & & $x$ & & & $\times$ \\
\hline 121 & 94 & Echinophyllia aspera (Ellis \& Solander) & & & $x$ & $\times$ & $x$ \\
\hline \multirow[t]{2}{*}{122} & & $\begin{array}{l}\text { Oxypora lacera (Verrill) } \\
\text { Dendrophylliidae }\end{array}$ & & $x$ & & & \\
\hline & 96 & $\begin{array}{l}\text { Turbinaria mesenterina (Lamarck) } \\
\text { Zod } \mathrm{n} \text { th a r i a } \\
\text { Zoanthidae }\end{array}$ & & & & & $x$ \\
\hline 123 & 97 & $\begin{array}{l}\text { Palythoa tuberculosa Esper } \\
\text { Antipat haria } \\
\text { Antipathidae }\end{array}$ & $x$ & & & & $x$ \\
\hline 124 & & Cirripathes sp. & & & $x$ & $x$ & \\
\hline
\end{tabular}


Tab. 2. Besiedlung der vier Testareale des Sanganeb-Atolls und des Testareals U-7 bei Aqaba durch die Cnidaria-Kategorien (", "

\begin{tabular}{|c|c|c|c|c|c|c|c|}
\hline \multirow{2}{*}{ Taxa } & \multicolumn{6}{|c|}{ Sanganeb-Testareale } & \multirow{2}{*}{$\begin{array}{c}\text { Aqaba- } \\
\text { Testarea } \\
\text { U-7 }\end{array}$} \\
\hline & I & II & III & IV & $\begin{array}{c}\text { Summe } \\
\text { I-IV }\end{array}$ & $\begin{array}{c}\text { Mittel- } \\
\text { wert }\end{array}$ & \\
\hline Hydroida & 2 & 3 & 3 & 4 & 12 & 3 & 1 \\
\hline Rhizostomeae & - & - & - & - & - & - & 1 \\
\hline Stolonifera & 1 & 1 & 1 & - & 3 & 0,75 & 1 \\
\hline Alcyonaria & 20 & 8 & 15 & 3 & 46 & 11,5 & 19 \\
\hline Gorgonaria & 1 & - & - & 1 & 2 & 0,5 & - \\
\hline Actiniaria & - & - & - & - & - & - & 3 \\
\hline Scleractinia & 44 & $55^{*}$ & 41 & 43 & $183^{*}$ & $45,75^{*}$ & $74^{*}$ \\
\hline Zoantharia & 1 & - & - & - & 1 & 0,25 & 1 \\
\hline Antipatharia & - & - & 1 & 1 & 2 & 0,5 & - \\
\hline Summe & 69 & $67^{\circ}$ & 61 & 52 & $249^{*}$ & $62,25^{\circ}$ & $100^{\cdots}$ \\
\hline
\end{tabular}

unterschiedlichem und meist geringem Ausmaß (vgl. Mergner \& Schuhmacher, 1981, 1985).

Wie viele Arten der verschiedenen Cnidaria-Gruppen in den einzelnen Testquadraten gefunden wurden, faßt Tabelle 2 zusammen. Beim Vergleich der Artenbestände aus den Testquadraten von Aqaba und dem Sanganeb-Atoll ist zu berücksichtigen, daß sich die Angaben für Aqaba auf $25 \mathrm{~m}^{2}$ beziehen, die für Sanganeb jedoch auf viermal $25 \mathrm{~m}^{2}$ mit jeweils unterschiedlicher Lage im Riff. Allein schon das Miteinbeziehen des Riffhanges bei Aqaba würde die Artenzahl aus dieser Region beträchtlich erhöhen. Für einen Vergleich mit dem Testareal U-7 bei Aqaba sollte daher die mittlere Artenzahl der Sanganeb-Quadrate herangezogen werden. Danach ergibt sich eine Artenvielfalt (pro $25 \mathrm{~m}^{2}$ ) von $62: 98$ zugunsten von Aqaba.

Das jeweils eigene Besiedlungsmuster der Sanganeb-Quadrate ist nach Mergner \& Schuhmacher (1985) hauptsächlich durch lokal wirkende hydrodynamische Einflüsse geprägt. Jedes einzelne Testquadrat unterscheidet sich erheblich vom Testareal U-7; am geringsten noch sind die Unterschiede in der Besiedlungsstruktur zwischen diesem und TQ I von der SW-Ecke des Sanganeb-Atolls. Um den Einfluß der Wasserbewegungsverhältnisse aus dem regionalen Vergleich herauszufiltern und damit den der temperaturbedingten Unterschiede herauszustellen, soll auch im folgenden das Testquadrat U-7 stets mit dem Mittelwert der vier Sanganeb-Quadrate verglichen werden.

Tabelle 3 bringt eine Übersicht über die wichtigsten Ergebnisse des Vergleiches zwischen den Testarealen von Aqaba und dem Sanganeb-Atoll. Sie betreffen vor allem Bedeckungsgrad, Koloniezahl und Koloniegröße, Diversitätsindex, Wuchsform und das Verhältnis von hermatypischen zu ahermatypischen Formen.

Insgesamt fanden sich im Testareal U-7 auf $25 \mathrm{~m}^{2}$ Fläche nach der Revision 98 Cnidaria-Arten (+ 2 Varietäten). Ihnen stehen in jedem Sanganeb-Areal durchschnittlich 62 Arten gegenüber. Bei Aqaba sind es also fast $63 \%$ mehr Arten pro Quadrat. Der Anteil riffbildender Steinkorallen an diesen Cnidaria betrug im U-7 34 Gattungen mit 72 Arten ( +2 Varietäten), aber in den vier Testquadraten des Sanganeb-Atolls zusammen auch nur 35 Gattungen mit 89 Arten $(+1$ Varietät); die Werte liegen pro Sanganeb- 
Tab. 3. Vergleich der Bedeckungsrate, Koloniezahl und KoloniegröBe, Diversität und Wuchstormen der Cnidaria zwischen den gemittelten Werten der vier Sanganeb-Testareale und dem AqabaAreal U-7

\begin{tabular}{|c|c|c|}
\hline Bodenbedeckung & $\begin{array}{c}\text { Sanganeb } \\
\text { TQ I-IV Mittelwerte }\end{array}$ & $\begin{array}{c}\text { Aqaba } \\
\text { Testareal U.7 }\end{array}$ \\
\hline Unbelebtes Substrat, Anteil an TQ $(\%)$ & 46,89 & 56,42 \\
\hline Begleitbenthos, Anteil an TQ (\%) & 2,78 & 0,37 \\
\hline Cnidaria-Bewuchs, Anteil an TQ (\%) & 50,21 & 42,28 \\
\hline Summe: Substrat + Lebendbewuchs (\%) & 99,88 & 99,07 \\
\hline \multicolumn{3}{|l|}{ Cnidaria: } \\
\hline Anzahl der Kolonien & 666,25 & 2051 \\
\hline Koloniegröße in $\mathrm{cm}^{2}$ & 189 & 52 \\
\hline Diversität $\mathrm{H}^{\prime}$ & 2,58 & 3,67 \\
\hline Eveness J & 0,64 & 0,80 \\
\hline \multicolumn{3}{|l|}{ Scleractinia, Wuchsformen } \\
\hline Verzweigte Arten, Anteil an TQ (\%) & 15.7 & 5,25 \\
\hline Massige Arten, Anteil an TQ (\%) & 14,4 & 10,02 \\
\hline Krustenförmige Arten, Anteil an TQ (\%) & 4,7 & 6,00 \\
\hline Hermatypische Arten, Anteil an TQ (\%) & 34,8 & 21,63 \\
\hline Ahermatypische Arten, Anteil an TQ (\%) & 15,4 & 20,62 \\
\hline
\end{tabular}

Areal zwischen 19/44 und 26/55 - im Mittel bei nur 22 Gattungen mit knapp 46 Arten. Analog zu der Situation bei den Cnideria allgemein übertrifft auch die Artendichte der Steinkorallen bei Aqaba diejenige am Sanganeb-Atoll um fast $64 \%$. Beiden Riffregionen, dem Testareal U-7 mit seinen 98 Cnidaria-Arten ( +2 Varietäten) wie auch den vier Sanganeb-Arealen mit ihren 123 Arten (+ 1 Varietät), waren 55 Arten (+ 1 Varietät) gemeinsam, nämlich Millepora exaesa, Tubipora musica, 9 Alcyonaria- und 43 Scleractinia-Arten (+ 1 Varietät) sowie Palythoa tuberculosa. Von diesen 55 Arten (+1) kamen im Sanganeb-TQ I 39 Arten vor (davon 27 Scleractinia), in TQ II $40+1$ (35), in TQ III 28 (20) und in TQ IV 21 (19).

Entsprechend der größeren Arten- und Koloniezahl auf gleich großer Fläche verändert sich von Süden nach Norden auch der Diversitätsindex: Auf die Koloniezahl bezogen, beträgt der Shannon-Wiener Index $\mathrm{H}^{\prime}$ bei Aqaba 3,67 und am Sanganeb-Atoll im Mittel 2,58 (2,34 bis 2,77). Im Testareal U-7 ist also die Artenvielfalt pro Fläche deutlich erhöht.

Bei einem Vergleich der Bestandsbildung in beiden Riffregionen (vgl. Mergner \& Schuhmacher, 1981, 1985) fällt auf, daß in den Testquadraten des Sanganeb-Atolls von 123 Cnidaria-Arten (+ 1 Varietät) immerhin 10 Arten (8\%) ansehnliche Bestände von annähernd einem Quadratmeter und darüber bilden, unter ihnen allein 3 Acropora- und 3 Alcyonaria-Arten. Im U-7 sind es dagegen nur 3 Alcyonaria-Arten (3\% des Artenbestandes). Kleinere Bestände mit etwa einem halben Quadratmeter Fläche finden sich im U-7 bei weiteren 6 Arten ( 1 Weichkoralle, 5 Steinkorallen) und am Sanganeb-Atoll bei weiteren 8 Arten ( 3 Weichkorallen, 5 Steinkorallen). Als Leitarten lassen sich im U-7 nur die beiden Xenia-Arten macrospiculata und umbellata mit ihren 232 und 202 Kolonien und Flächen von 1,42 und $1,28 \mathrm{~m}^{2}$ bezeichnen. Daneben bringt es auch Goniastrea 
pectinata auf 162 Kolonien, aber nur auf 0,42 $\mathrm{m}^{2}$ Fläche. Alle anderen Arten liegen entweder bei der Koloniezahl oder der Siedlungsfläche weit unter diesen Werten.

Demgegenüber treten in den Sanganeb-Testarealen II bis IV eindeutige Leitarten hervor, die zugleich auch ausgeprägte biophysiographische Zonen (zur Definition vgl. Mergner \& Schuhmacher, 1974) bilden. Beim Testquadrat U-7 kann dagegen keine irgendwie geartete Zone charakterisiert werden, ähnlich wie bei TQ I am SanganebAtoll, obwohl auch hier Xenia macrospiculata mit 216 Kolonien auf 3,9 $\mathrm{m}^{2}$ Fläche einen ausgedehnten, aber in sich nicht geschlossenen Bestand aufweist. Wie weiter oben schon erwähnt, sind diese beiden Testareale, U-7 bei Aqaba und TQ I am SanganebAtoll, noch am ehesten miteinander vergleichbar: Im Bereich ruhiger Wasserbewegung gelegen, zeigen nämlich beide Areale die höchste Anzahl an Arten und Kolonien mit nahezu gleichen Anteilen von Stein- und Weichkorallen, wobei Xenia macrospiculata die Leitart darstellt. Unter den Steinkorallen herrschen solche mit massigem Wuchs leicht vor, und unbelebtes Substrat macht relativ große Flächen aus (U-7: $56 \%$, TQ I: $53 \%$ ). Es kann hier darauf hingewiesen werden, daß die große Anzahl von XeniaKolonien nur durch die eingehende in situ-Untersuchung deutlich wurde; semi-quantitative Schätzmethoden (z. B. Maragos, 1974) hätten die auf den ersten Blick unscheinba* ren Bestände unterrepräsentiert.

Natürlich können die Zunahme der Arten- und Koloniezahl pro Fläche und damit die Vergrößerung der Artenvielfalt pro Bezugsfläche nur auf Kosten des unbelebten Restareals oder der Koloniegröße erfolgen. Da aber der Anteil des unbelebten Substrates (abgestorbener Korallenfels und Sandboden) im Testareal U-7 mit 14,2 $\mathrm{m}^{2}(=56,4 \%$ ) von $25 \mathrm{~m}^{2}$ größer ist als der durchschnittliche Anteil am Sanganeb-Atoll mit $11,73 \mathrm{~m}^{2}(=$ $46,9 \%)$, kann sich die Artenvielfalt nur zu Lasten der Koloniegröße vergrößert haben: So beträgt letztere am Sanganeb-Atoll im Mittel $189 \mathrm{~cm}^{2}$, im U-7 jedoch nur $52 \mathrm{~cm}^{2}$, also weit weniger als ein Drittel - das allerdings bei mehr als dreifacher Koloniezahl; denn im Mittel wurden pro Sanganeb-Quadrat 662 Kolonien gezählt, im U-7 dagegen 2028. Bei den beiden wichtigsten Kategorien der Cnidaria herrschen vergleichbare Verhältnisse: Von den Alcyonaria stehen bei Aqaba 749 Kolonien auf durchschnittlich je $69 \mathrm{~cm}^{2}$ Fläche den im Mittel 260 Kolonien am Sanganeb mit je $144 \mathrm{~cm}^{2}$ Fläche gegenüber. Bei den Scleractinia sind es bei Aqaba 1247 Kolonien mit einer Durchschnittsgröße von 42 $\mathrm{cm}^{2}$ Fläche und am Sanganeb-Atoll 336 Kolonien mit je $243 \mathrm{~cm}^{2}$. Bei den Steinkorallen ist also die durchschnittliche Koloniefläche am Sanganeb-Atoll sechsmal größer als bei Aqaba, bei den Weichkorallen reichlich zweimal.

Von besonderem Interesse ist dabei die Frage, ob nur bestimmte Arten der Weichund Steinkorallen in einer der beiden Riffregionen durchschnittlich größere Kolonien bilden oder auf die unterschiedlichen Lebensverhältnisse mit unterschiedlichen Koloniezahlen reagieren. Hierzu sollen von den gemeinsam vorkommenden 9 AlcyonariaArten 4 zur Überprüfung herangezogen werden, von den 43 Scleractinia-Arten $(+1$ Var.) entsprechend 21 Arten, hingegen bleiben Arten mit zu geringen vergleichbaren Beständen unberücksichtigt. Tabelle 4 vergleicht Koloniegröße und Kolonieanzahl zwischen dem Testareal U-7 (jeweils linke Zahlenkolonne) und den Durchschnittswerten der vier Sanganeb-Areale (rechte Zahlenkolonne). Die nicht berücksichtigten Arten mit zu geringen Koloniezahlen lagen durchweg auf der Linie der sich in Tabelle 4 abzeichnenden Tendenz:

Im Verhältnis zwischen der Anzahl der Kolonien im Testareal U-7 und der mittleren 
Tab. 4. Vergleich der Koloniegröße und Kolonieanzahl zwischen dem Testareal U-7 bei Aqaba (jeweils linke Zahlenkolonne) und den Mittelwerten der vier Sanganeb-Areale (jeweils rechte Zahlenkolonne)

\begin{tabular}{|c|c|c|}
\hline \multirow[t]{2}{*}{ Species } & \multicolumn{2}{|c|}{ Mittelwerte } \\
\hline & $\begin{array}{c}\text { Koloniegröße } \\
\text { U-7 : I-IV }\end{array}$ & $\begin{array}{c}\text { Kolonieanzahl } \\
\text { U-7 : I-IV }\end{array}$ \\
\hline Parerythropodium fulvum (7) & $1: 2,26$ & $3,87: 1$ \\
\hline Sarcophyton ehrenbergi (8) & $1,92: 1$ & $2,66: 1$ \\
\hline Xenia macrospiculata (26) & $1: 2,15$ & $2,28: 1$ \\
\hline Xenia umbellata (27) & $1,54: 1$ & $25,25: 1$ \\
\hline Stylophora pistillata (36) & $1: 1,38$ & $11,39: 1$ \\
\hline Seriatopora hystrix (38) & $1: 2,25$ & $1: 2,75$ \\
\hline Acropora hemprichi (45) & $1: 14,53$ & $1: 2,08$ \\
\hline Acropora humilis (46) & $: 8,11$ & $: 1$ \\
\hline Acropora variabilis (51) & $: 3,55$ & $6,74: 1$ \\
\hline Montipora meandrina (56) & $1: 5,38$ & $31,00: 1$ \\
\hline Montipora venosa $(60)$ & $: 1,46$ & $2,67: 1$ \\
\hline Pavona varians $(67)$ & $1: 1,93$ & $3,22: 1$ \\
\hline Porites lutea (80) & $: 5,95$ & $1: 1,75$ \\
\hline Favia laxa (86) & $: 2,47$ & $5,60: 1$ \\
\hline Favia stelligera (90) & $: 7,70$ & $2,40: 1$ \\
\hline Goniastrea pectinata (97) & $: 3,06$ & $9,82: 1$ \\
\hline Goniastrea retiformis (98) & : 5,12 & $6,35: 1$ \\
\hline Leptastrea bottae (103) & $: 5,33$ & $38,67: 1$ \\
\hline Leptastrea transversa (105) & $: 3,55$ & $11,00: 1$ \\
\hline Cyphastrea chalcidicum (106) & $: 2,67$ & $1,88: 1$ \\
\hline Cyphastrea microphthalma (107) & $: 1,97$ & $27,00: 1$ \\
\hline Echinopora gemmacea (109) & $: 7,75$ & $1,71: 1$ \\
\hline var. fruticulosa (110) & $: 13,71$ & $6,40 \div 1$ \\
\hline Galaxea fascicularis (113) & $: 5,63$ & $1: 1,17$ \\
\hline Lobophyllia corymbosa (115) & $: 20,26$ & $1: 10,75$ \\
\hline
\end{tabular}

Anzahl aus den vier Sanganeb-Arealen überwiegt fast durchweg die am U-7 ermittelte Koloniezahl. Sie übertrifft teilweise, so bei Xenia umbellata, Montipora meandrina, Leptastrea bottae und Cyphastrea microphthalma, die Werte vom Sanganeb-Atoll um mehr als das Zwanzigfache. Nur wenige Arten, insgesamt 5 von 25, sind dort wiederum häufiger vertreten, meist nur bis zum Dreifachen. Lediglich Lobophyllia corymbosa macht hierin eine Ausnahme und ist am Sanganeb-Atoll fast elfmal häufiger als im U-7. Da Lobophyllia offensichtlich strömendes Wasser bevorzugt und stärkere Vertikalbewegung meidet, kommt sie auch von den Sanganeb-Quadraten fast nur im TQ II vor, dort allerdings dann mehr als vierzigmal so häufig als im U-7.

In der linken Spalte von Tabelle 4 stehen sich die Koloniegrößen von U-7 und den Mittelwerten aus den vier Sanganeb-Arealen gegenüber. Wiederum zeigt sich ein deutlicher Unterschied zwischen den beiden Riffregionen: Mit Ausnahme von nur zwei Alcyonaria-Arten (Sarcophyton ehrenbergi, Xenia umbellata) sind die Kolonien gleicher Arten am Sanganeb-Atoll deutlich größer als im U-7.

Besonders auffällig ist die offensichtlich durch die günstigen Umweltbedingungen gesteigerte Wüchsigkeit bei den verzweigten Acropora hemprichi, A. humilis, Echino- 
pora gemmacea var. fruticulosa und der krustenförmig wachsenden Normalform $E$. gemmacea, sowie bei den massigen Favia stelligera und Lobophyllia corymbosa. Von diesen treten $A$. hemprichi und $L$. corymbosa in den Sanganeb-Arealen sowohl durch erheblich größere Koloniezahl als auch durch bedeutendere Koloniegröße gegenüber dem Aqaba-Areal hervor. Beide Arten werden offensichtlich durch den im Vergleich zu Aqaba wesentlich intensiveren Wasseraustausch begünstigt (vgl. Mergner \& Schuhmacher, 1985).

\section{DISKUSSION}

Nach Scheer (1984) zählt das mittlere Rote Meer zu den Zentren der Korallenvielfalt im Indopazifik. Ob diese zum nördlichen Golf von Aqaba, dem äußersten Vorposten des Riffgürtels im Bereich des Indischen Ozeans, merklich reduziert ist, darauf gibt der Vergleich der Artenbestände riffbildender Steinkorallen (hinsichtlich Weichkorallen liegen keine entsprechenden Daten vor) keine eindeutige Antwort. Wie einleitend ausgeführt wurde, ist der zur Zeit bekannte Artenbestand an Riffkorallen im ausgedehnten Riffgebiet vor der sudanesischen Küste unwesentlich höher als am Nordende des Golfes von Aqaba (51 : 48 Gattungen mit jeweils ungefähr 120-130 Arten). Welche Rückschlüsse ergeben sich dann aus der flächenbezogenen quantitativen Analyse des Korallenbestandes aus beiden Regionen?

Der Vergleich des Aqaba-Quadrates mit dem "gemittelten" Sanganeb-Quadrat zeigt für das Nordende des Roten Meeres eine höhere Artendichte, Koloniezahl und damit Diversität pro Bezugsfläche, aber eine geringere Koloniegröße als im mittleren Roten Meer (Tab. 3 und 4). Im Aqaba-Testareal U-7 wurden 2059 Kolonien von 98 Arten (+2 Varietäten) (104 Arten vor der Revision) mit einem Diversitätsindex hinsichtlich der Koloniezahl von $\mathrm{H}^{\prime}=3,67$ festgestellt (Mergner \& Schuhmacher, 1981). Dieser Index stellt einen aus Korallenriffbiotopen bisher noch nicht erhobenen Höchstwert dar. Die für die Sanganeb-Quadrate ermittelten Indices betragen demgegenüber im Mittel 2,58 $(2,34$ bis 2,77$)$. Das bedeutet, daß vom mittleren Roten Meer mit seinen optimalen Entwicklungsbedingungen für Riffkorallen zum Nordende des Meeres mit nur noch suboptimalen Bedingungen (nahe der Existenzgrenze für Riffkorallen) die Artendichte, Koloniezahl und -diversität nicht fallen, sondern sogar ansteigen. Bei diesem Vergleich ist allerdings zu berücksichtigen, daß der Shannon-Wiener Index rein numerisch mit der absoluten Artenzahl steigt (Webb, 1974); daher sollte der Aqaba-Wert, der auf einer um $63 \%$ höheren Artenzahl als der Sanganeb-Wert beruht, nicht überinterpretiert werden.

Beim Vergleich der Eveness J (nach Pielou, 1966) ergibt sich ein ähnliches Bild. Der Wert liegt für Aqaba mit $\mathrm{J}=0,80$ deutlich über dem entsprechenden Mittelwert von Sanganeb mit 0,64 (0,56 bis 0,70). Da sich Pielous Formel zur Erfassung der Eveness auf den Shannon-Wiener Index stützt, ist allerdings auch hier eine direkte Relation zwischen dem Wert $J$ und dem zugrunde liegenden Artenumfang gegeben (vgl. Alatalo, 1981).

Korallenwachstum ist temperaturabhängig (z. B. Weber \& White, 1974). Unter den günstigen Temperaturbedingungen des mittleren Roten Meeres, dessen Jahresmittel $5{ }^{\circ} \mathrm{C}$ über dem des nördlichen Golfes von Aqaba liegt (Abb, 1), können raschwüchsige oder auf andere Weise konkurrenzstarke Arten große Kolonien und ausgedehnte Bestände bilden. Pro Flächeneinheit vermindert sich damit zwangsläufig die Artenzahl und-diversität. Aber auch bei verlangsamtem Wachstum, wie es bei Aqaba offensicht- 
lich der Fall ist, sollte es am Ende zur Dominanz relativ weniger, bestangepaßter Arten kommen. Wenn dieser Zustand offenbar nicht erreicht wird, sondern bei anscheinend reichlich Siedlungsfläche (ca. 30\% Fläche ist unbewohnter Korallenfels) ein breites Spektrum von Cnidaria Fuß faßt, muß nach Gründen gefragt werden, die das Zustandekommen einer wenig diversen Gemeinschaft standortangepaßter Arten verhindert.

Von den verschiedenen Hypothesen, die Gesetzmäßigkeiten in der Entwicklung von Diversitätsmustern aufzudecken suchen, ist zunächst die , intermediate disturbance hypothesis" (Connell, 1978) zu nennen. Hier wird aufgezeigt, wie eine Gemeinschaft in ihrer Sukzession auf ein wenig diverses Endstadium hin beständig durch Störungen und Katastrophen zurückgeworfen und damit langfristig in der relativ hoch diversen Zwischenphase zwischen Pionier- und Endstadium gehalten wird. Connell führte das Korallenriff und den tropischen Regenwald als Belegbeispiele für seine Hypothese an.

Welche Störungen wirken nun auf das Testquadrat U-7 bei Aqaba ein und halten seine Diversität hoch? Die Temperatur ist zwar im Winter mit etwa $21^{\circ} \mathrm{C}$ niedrig, aber doch beständig über dem Existenzminimum von Riffkorallen. Denn das Wasser im Golf wird ständig umgewälzt und ist dabei bis in die Tiefe homogenisiert - bis $1800 \mathrm{~m}$ fällt die Temperatur nicht unter $20,9^{\circ} \mathrm{C}$ (Klinker et al., 1976). Flache Rifflagunen können zwar im Winter auf $15^{\circ} \mathrm{C}$ (Mergner \& Svoboda, 1977) abkühlen - und dieses Wasser verhindert offensichtlich auch Korallenaufwuchs in den canyonartigen Rücklaufkanälen des Lagunensaumriffes südlich des hier in Rede stehenden Untersuchungsgebietes (Mergner \& Schuhmacher, 1974) - es ist aber auszuschließen, daß derart abgekühltes Wasser noch das Testquadrat U-7 in $10 \mathrm{~m}$ Tiefe $70 \mathrm{~m}$ vor der Riffkante erreicht.

Eine Gefährdung der Korallen im Areal U-7 liegt allerdings in der nur schwach geneigten Lage des Areals im noch mäBig abfallenden mittleren Vorriff begründet. Die Sanganeb-Areale befinden sich hingegen alle auf einem mehr oder weniger steilen Riffhang. Gelegentlich auftretende Sedimentbelastungen - im Quadrat U-7 durch Sturmbewegungen aufgewirbelter Sand aus den umliegenden Vertiefungen und durch Strömung verfrachteter Sand von nahegelegenen Hafenbauten - können die Kolonien nachhaltig schädigen. „Glatzenartig“ abgestorbene Scheitel massiger Kolonieformen weisen hierauf hin (Abb. 4). Der kahle Korallenfels ermöglicht Algenaufwuchs, der Diademseeigeln als Weide dient. Wohl nicht zuletzt aufgrund des guten Nahrungsangebotes und auch wegen der flachen Erstreckung des Testquadrates U-7 war die Individuendichte von Diadema setosum hier um ein Mehrfaches größer als in den SanganebQuadraten. Dort kann neben der relativ steilen Lage der Quadrate auch ihre teilweise Exposition zu heftiger Wasserbewegung eine nennenswerte Diadema setosum-Besiedlung verhindert haben. Wie bei Mergner \& Schuhmacher (1981) schon ausgeführt, sind ältere Kolonien nicht selten durch abgestorbene Stellen in einzelne, selbständig weiterwachsende Fragmente unterteilt. Dazwischen können sich neue andere Kolonien angesiedelt haben (Abb. 4). Die Fragmente mußten in der diesem Vergleich zugrunde liegenden Erhebung als getrennte Kolonien gezählt werden; sie haben zusätzlich zu dem hohen Diversitätsindex beigetragen.

Die Darlegung der lokalen Situation von Testquadrat U-7 unterstützt die Erklärung, warum immer wieder Platz für die Ansiedlung einer großen Artenvielfalt bereitsteht und warum die Koloniezahl hoch, die Koloniegröße jedoch klein ist. Die Frage bleibt jedoch, ob nicht die Sanganeb-Quadrate prinzipiell denselben Risiken unterworfen sind.

Auch sie weisen einen unbesiedelten Hartbodenanteil von $38,83 \%$ (TQ II) bis 


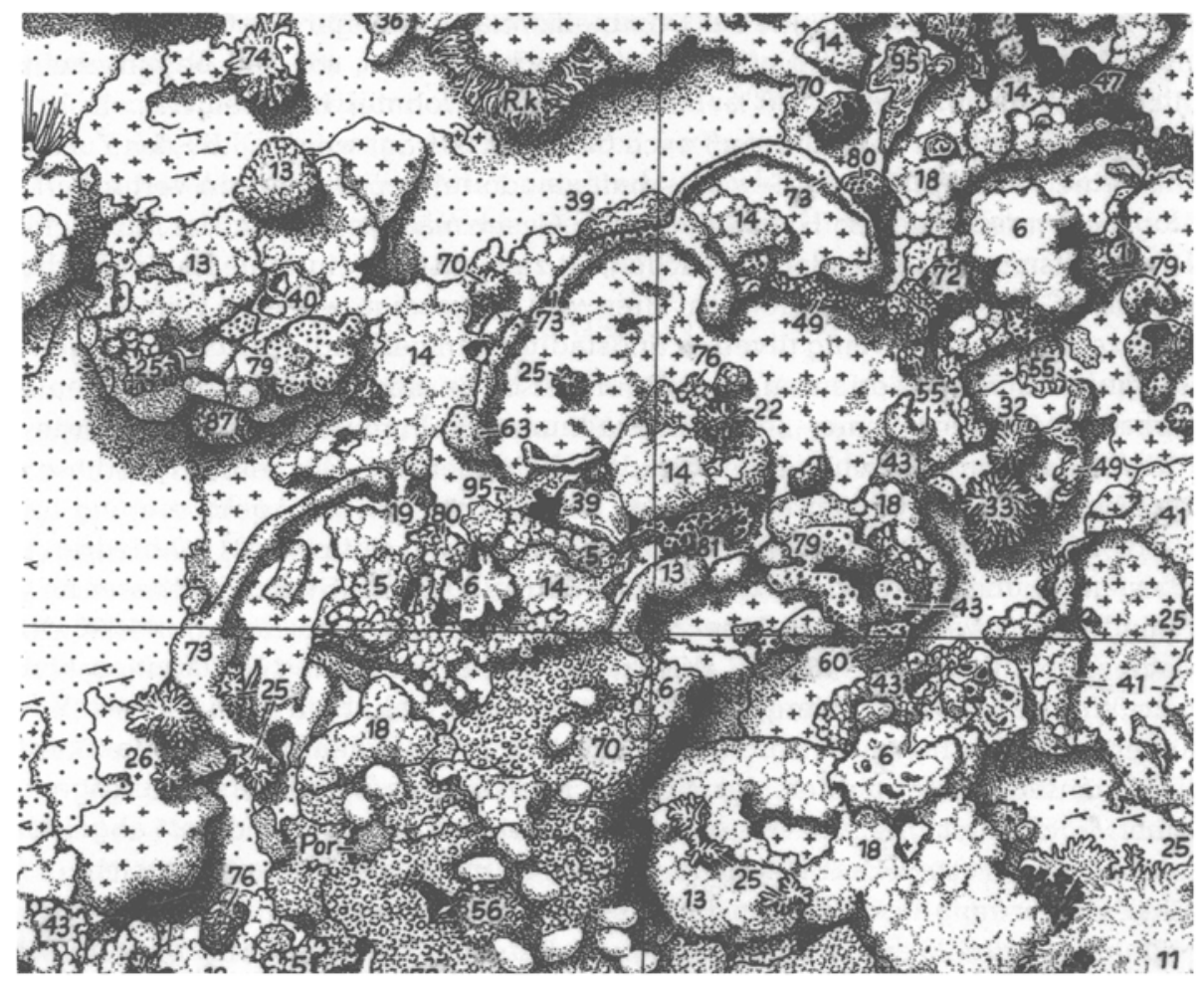

Abb. 4. Ausschnitt aus dem Testareal U-7 bei Aqaba (Einzelquadrate Ild und IIId) mit oberseitig durch Sedimentation und Diadema-Beweidung abgestorbener Kolonie von Favia stelligera (Nr. 73).

Die mikroatollartigen lebenden Randpartien entwickeln sich als einzelne Kolonien weiter

$45,73 \%$ (TQ I) auf. Anstelle von Diademseeigeln weiden vornehmlich herbivore Fische wie Pomacentrus lividus und Acanthurus sohal (Vine, 1974) den Algenaufwuchs ab. Bei TQ I, das auf der Leeseite eines breiten Riffdaches liegt, kann erwartet werden, daß gelegentlich bei Sturm erhebliches Sedimentmaterial vom Riffdach aufgewirbelt und auf die Testquadratflächen abgelagert wird. Bei TQ III, ebenfalls im Lee des Riffdaches gelegen, ist dies jedoch wegen der senkrechten bis überhängenden Arealfläche nicht möglich. Tatsächlich waren entsprechende Hinweise auch nicht festzustellen. Die hydrodynamisch exponierten Quadrate, besonders TQ IV, erleiden sicher bei Sturmbrandungen Schäden. Da bei unserer Untersuchung allerdings Spuren einer besonders heftigen Störeinwirkung nicht zu bemerken waren, ist anzunehmen, daß Schäden im Rahmen bleiben und rasch wieder durch Regeneration und Neuansiedlung ausgeglichen werden.

Weichkorallen behindern den Aufwuchs von Steinkorallen. Sie schatten die Steinkorallenpolypen von Licht- und Wasserströmung $a b$, bringen sie zum Absterben und überziehen das tote Skelett (Schuhmacher, 1975). Besonders Xenia-Rasen können sich zu Lasten von Steinkorallen rasch ausdehnen, die Kolonien sterben dann aber - ohne erkennbaren Grund - ebenfalls ab, verschwinden völlig und hinterlassen kahlen Korallenfels. 
Je nach Standort sind es also andere Einflüsse, welche die Entwicklung zu einer von wenigen Arten beherrschten Gemeinschaft aufhalten. Das Besetzen freier Flächen durch die Siedlungsgemeinschaft erfolgt allerdings bei Aqaba und Sanganeb unterschiedlich schnell. Hier spielt nun doch die unterschiedliche Wassertemperatur eine Rolle. Wegen der Temperaturabhängigkeit des Korallenwachstums können Regeneration und Wiederbesiedlung langsamer oder schneller erfolgen. Unter der Annahme, daß Rückschläge in der Sukzession zum Endzustand mit gleicher Häufigkeit auftreten, sollte die geringere Regenerationsgeschwindigkeit bei Aqaba eine längere Phase höherer Diversität bedingen als im mittleren Roten Meer, wo eine solche Phase durch das relativ rasche Ausbreiten angepaßter Arten eher begrenzt wird. Wenn nun - was sehr wahrscheinlich ist - die Häufigkeit (unvorhersehbarer) widriger Einflüsse auf die Korallengemeinschaft im nördlichen Golf von Aqqaba größer als im mittleren Roten Meer ist, wird in ersterem eine höhere Diversität beständig aufrecht erhalten.

Auch das "dynamic equilibrium model" von Huston (1979) sieht dann die höchste Diversität vor, wenn eine Gemeinschaft noch nicht einen durch interspezifische Konkurrenz bestimmten Gleichgewichtszustand erreicht hat. Ca, 30-45\% freier, potentiell besiedelbarer Korallenfels in beiden Untersuchungsgebieten deuten zwar darauf hin, daß zumindest theoretisch die Kapazitätsgrenze der Testflächen noch nicht erreicht ist. Tatsächlich erfordert aber der ständige Umschlag der Bestände, z. B. infolge abiotischer Faktoren, Diadema-Überweidung, Xenia-Verdrängung, notwendigerweise einen gewissen Flächenspielraum. Dabei werden freie Areale durch Diadema-Einfluß sicher länger offen gehalten als infolge vorübergehender Xenia-Überwachsung. Diese Dynamik kann allerdings nur durch eine Langzeituntersuchung erfaßt werden. Nach Hustons für Korallenriffe noch einmal speziell dargelegten Hypothese (1985) wird die Diversität entscheidend durch das Verhältnis zwischen Häufigkeit von Störeinwirkungen und Wachstumsrate der betroffenen Korallen bestimmt. Der Vergleich zwischen Aqaba und Sanganeb belegt nun Hustons Postulat, daß langsames Wachstum das Stadium "drükkender" und damit Artendiversität reduzierender Raumkonkurrenz auf einen (ggf. unendlich) späten Zeitpunkt verschiebt.

Loya (1972) fand, daß zwischen Riffdach und $40 \mathrm{~m}$ Tiefe Artenzahl und Diversität der hermatypischen Steinkorallen mit der Tiefe zunahmen. Offensichtlich erlaubt die unter suboptimalen Lichtbedingungen verringerte interspezifische Konkurrenz das Nebeneinander von mehr Arten als unter optimalen Lichtbedingungen. Artenzahl und Diversität sinken erst bei Annäherung an die lichtbedingte Existenzgrenze der Zooxanthellen-haltigen Korallen, die bei manchen Arten erst in ca. $100 \mathrm{~m}$ Tiefe liegt (Fricke \& Schuhmacher, 1983). Analog zur bathymetrischen Verteilung von Artenzahl und Diversität ist das hier untersuchte latitudinale Verbreitungsmuster der Riffkorallen zu sehen. Statt Lichtangebot ist die Temperatur der variierte Faktor. In beiden Fällen wird unter suboptimalen Bedingungen Artenvielfalt und Diversität gefördert.

Eine Generalisierung der vorliegenden Befunde lautet etwa so: Im Roten Meer geht mit der Verschlechterung der Temperaturbedingungen zum nördlichen Randbereich hin eine Zunahme der flächenbezogenen Artenvielfalt und Diversität der Korallen einher, während Temperaturerhöhung und andere Verbesserungen ihrer Lebensbedingungen gegen das Verbreitungszentrum hin das Dominieren von relativ wenigen Arten fördern.

Diese Hypothese ist in anderen Riffgebieten noch auf ihre Allgemeingültigkeit zu 
überprüfen. Sie muß nicht der bekannten Vorstellung einer Abnahme der Artenzahl der Riffkorallen gegen den Rand ihres Verbreitungsgebietes widersprechen. Rosen (1981) führt für den Indischen Ozean aus, daß Zooxanthellen-haltige Korallen bei einer geographischen Breite von ca. $30^{\circ} \mathrm{N}$ und $30^{\circ} \mathrm{S}$ einen "steep diversity rise" zeigen, der sich gedämpft, aber mehr oder weniger stetig, äquatorwärts fortsetzt. Diese grobe, nämlich nur auf absoluten Gattungszahlen beruhende Abschätzung der Diversitätszunahme trifft (in abgeschwächter Form) auch auf das Rote Meer zwischen $29^{\circ} 30^{\prime}$ und $20^{\circ} \mathrm{N}$ zu. Sie sagt aber nichts über die Artenzahl pro Fläche und ihre Aquität (evenness) aus.

Die hohe Artenzahl und Diversität der Riffkorallen bei Aqaba weisen ferner darauf hin, daß am Nordende des Golfes von Aqaba die physiologische Existenzgrenze für das Gros der Rotmeer-Korallen noch nicht erreicht ist. Würde sich der Golf weiter nach Norden fortsetzen, würde die temperaturbedingte Grenze des Riffvorkommens sicherlich nördlich von Eilat und Aqaba liegen. Damit unterscheidet sich der nördlichste Riffvorposten deutlich von seinen südlichen Pendants im Indischen Ozean. Inhaca Island (bei $26^{\circ} \mathrm{S}$ vor der ostafrikanischen Küste) gilt als südlichstes Riffvorkommen im westlichen Indischen Ozean. Hier wurden noch 35 Gattungen von Riffkorallen festgestellt (MacNae \& Kalk, 1969). Die südlichsten Riffe des Indischen Ozeans überhaupt befinden sich allerdings auf dem westaustralischen Schelf bei den Houtman AbrolhosInseln. Dieser Archipel zwischen $28^{\circ}$ und $29^{\circ} \mathrm{S}$ ( $60 \mathrm{~km}$ vor der Küste) beherbergt noch 37 Gattungen von Riffkorallen (Wilson \& Marsh, 1980; Johannes et al., 1983), während auf ungefähr gleicher geographischer Länge vor SW-Sulawesi (Celebes) bei $5^{\circ} \mathrm{S} 78$ Gattungen und Untergattungen erfaßt worden sind (Moll, 1983). Die Wassertemperatur variiert bei den Houtman Albrolhos-Inseln im Jahr zwischen $26^{\circ}$ und $18^{\circ} \mathrm{C}$. Hier ist die Grenze des Riffvorkommens tatsächlich temperaturbedingt. Der Vergleich der beiden RiffauBenposten bestätigt auch, daß der biogeographische Äquator nordwärts verschoben ist (Böhnecke, 1936; zit. in Stehli \& Wells, 1971).

In den Benthosgemeinschaften von Houtman Abrolhos geben die Korallen ihre dominierende Stellung an Makroalgen ab (z. B. Ecklonia radiata). Trotz oder - im Licht der vorangegangenen Diskussion - wahrscheinlich auch wegen der Konkurrenz von Tangen stellen Johannes et al. (1983) fest: "Coral diversity is exceptionally high". Diese zunächst qualitative Aussage sollte durch eine quantitative Analyse untermauert werden. Mit ihr ergäbe sich eine hervorragende Gelegenheit, die Besiedlungsstruktur der Riffgemeinschaften in dieser Randlage mit solchen weiter nördlich zu vergleichen und neben die vorliegende Vergleichsuntersuchung aus dem Roten Meer zu stellen. Denn zur weiteren Untersuchung der Randlagebedingungen ist ein zusammenhängendes Riffgebiet in Nordsüd-Erstreckung mit durchgängigen Faktorengradienten besser geeignet als isolierte Riffaußenposten, wie Bermuda und die Hawaii-Inseln, bei denen eine durch die Insellage bedingte Artenverarmung zusätzlich zu berücksichtigen wäre.

Danksagungen. Wir danken der Deutschen Forschungsgemeinschaft für die finanzielle Unterstützung unseres Sanganeb-Projektes, unseren beiden Mitarbeitern cand.rer.nat. F. Nobbe und L. Schillak (Ruhr-Universität Bochum) für ihren engagierten Einsatz bei der Durchführung der Forschungsreise, bei der Erfassung der ökologischen Daten und Belegstücke sowie bei der ständigen Wartung des Gerätes, ferner dem National Council for Research and Technical Development, Khartoum, für die Genehmigung unserer Untersuchungen und der erforderlichen Aufsammlungen, dem Direktor des Institute of Oceanography Port Sudan, Professor Dr. J. Schroeder, und seinen Mitarbeitern für ihre stetige Hilfsbereitschaft, und der Besatzung des Sanganeb-Leuchtturmes für 
ihre Gastfreundschaft. Bei der Auswertung der Untersuchungsergebnisse haben in dankenswerter Weise Herr H. Löseke durch Erstellen der Datentabellen mitgewirkt. Schließlich möchten wir den Kollegen unseren besonderen Dank übermitteln, die taxonomisch schwierige Belegexemplare bestimmten oder unsere Determinationen überprüften: Dr. J. Verseveldt (Alcyonaria), Dr. G. Scheer und Dr. M. Wijsman-Best (Scleractinia). Ein unbekannter Gutachter bereicherte die Diskussion durch wertvolle Hinweise.

\section{LITERATUR}

Alatalo, R. V., 1981. Problems in the measurement of eveness in ecology. - Oikos 37, $199-204$.

Barnes, D. J. \& Taylor, D. L., 1973. In situ studies of calcification and photosynthetic carbon fixation in the coral Montastrea annularis. - Helgoländer wiss. Meeresunters. 24, 284-291.

Connell, J. H., 1978. Diversity in tropical rain forests and coral reefs. - Science, N. Y. 199, $1302-1310$

Crossland, C., 1907. Reports on the marine biology of the Sudanese Red Sea. IV. The recent history of the coral reefs of the mid-west shores of the Red Sea. - J. Linn. Soc. 31, 14-30.

Crossland, $C_{*}$ 1935. Coral faunas of the Red Sea and Tahiti. - Proc. zool. Soc. Lond. 1935, $499-504$.

Crossland, C., 1938. The coral reefs at Ghardaqa, Red Sea. - Proc. Zool. Soc. Lond. (A) 108, 513-521.

Crossland, C., 1939. Reports on the preliminary expedition for the exploration of the Red Sea. I. Narrative and list of stations. - Publs mar. biol. Stn. Ghardaqa 1, 3-11.

Ehrenberg, C. G., 1834a. Beiträge zur physiologischen Kenntniss der Corallenthiere im allgemeinen, und besonders des rothen Meeres, nebst einem Versuch zur physiologischen Systematik derselben. - Phys. Abh. Kgl. Akad. Wiss. Berlin 1832, 225-380.

Ehrenberg, C. G., 1834b. Über die Natur und Bildung der Corallenbänke des rothen Meeres. - Phys. Abh. Kgl. Akad. Wiss. Berlin 1832, 381-432.

Forskål, P., 1775. Descriptiones animalium, avium, amphibiorum, piscium, insectorum, vermium, quae in itinere orientali observavit Petrus Forskal. Ed.: Carsten Niebuhr.

Fricke, H. W. \& Schuhmacher, H., 1983. The depth limits of Red Sea stony corals: an ecophysiological problem. - Mar. Ecol. 4, 163-194.

Glynn, P. W., 1973. Aspects of the ecology of coral reefs in the Western Atlantic Region. In: Biology and geology of coral reefs. Ed, by O. A. Jones \& R. Endean. Acad, Press, New York, 1, 271-324.

Head, S. M., 1980. The ecology of corals in the Sudanese Red Sea. Thesis, Cambridge Univ., 471 pp.

Huston, M, 1979. A general hypothesis of species diversity. - Am. Nat. 113, 81-101.

Huston, M., 1985. Patterns of species diversity on coral reefs. - A. Rev. Ecol. Syst. 16, $149-177$.

Hulings, N. C., 1979. Currents in the Jordan Gulf of Aqaba. - Dirasat 6, 21-33.

Johannes, R. E., Wiebe, W. J., Crossland, C. J., Rimmer, D. W. \& Smith, S. V., 1983. Latitudinal limits of coral reef growth. - Mar. Ecol. Prog. Ser. 11, 105-111.

Klinker, J., Reiss, Z., Kropach, C., Levanon, I., Harpaz, H., Halicz, E. \& Assaf, G., 1976. Observations on the circulation pattern in the Gulf of Eilat (Aqaba), Red Sea. - Israel J. Earth Sci. 25, 85-103.

Klunzinger, C. B., 1872. Zoologische Excursion auf ein Korallenriff des Rothen Meeres bei Kossér. Z. Ges. Erdk. Berlin 7, 20-56.

Klunzinger, C. B., 1877. Die Korallthiere des Rothen Meeres, 1: Die Alcyonarien und Malacodermen. Gutmann, Berlin, $98 \mathrm{pp}$.

Klunzinger, C. B., 1879a. Die Korallthiere des Rothen Meeres. 2: Die Steinkorallen. 1: Madreporaceen und Oculinaceen. Gutmann, Berlin, $88 \mathrm{pp}$.

Klunzinger, C. B., 1879b. Die Korallthiere des Rothen Meeres. 3: Die Steinkorallen. 2: Astraeaceen und Fungiaceen. Gutmann, Berlin, $100 \mathrm{pp}$.

Kühlmann, D., 1983. The composition and ecology of deep-water coral associations. - Helgoländer Meeresunters. 36, 183-204.

Loya, Y., 1972. Community structure and species diversity of hermatypic corals at Eilat, Red Sea. Mar. Biol. 13, 100-123.

Loya, Y. \& Slobodkin, L. B., 1971. The coral reefs of Eilat (Gulf of Eilat, Red Sea). - Symp. zool. Soc. Lond. 28, 117-139.

Macnae, W. \& Kalk, M., 1969. A natural history of Inhaca Island, Moçambique. Witwatersrand Univ. Press, Johannesburg, 163 pp. 
Maragos, J. E., 1974. Coral communities on a seaward reef slope, Fanning Island. - Pacif, Sci. 28, $257-278$.

Mergner, H., 1984. The ecological research on coral reefs of the Red Sea. - Deep Sea Res. (A) 31, 855-884.

Mergner, H. \& Schuhmacher, H., 1974. Morphologie, Okologie und Zonierung von Korallenriffen bei Aqaba (Golf von Aqaba, Rotes Meer). - Helgoländer wiss. Meeresunters. 26, 238-358.

Mergner, H. \& Schuhmacher, H., 1981. Quantitative Analyse der Korallenbesiedlung eines Vorriffareals bei Aqaba (Rotes Meer). - Helgoländer Meeresunters. 34, 337-354.

Mergner, H. \& Schuhmacher, H., 1985. Quantitative Analyse von Korallengemeinschaften des Sanganeb-Atolls (mittleres Rotes Meer) I. Die Besiedlungsstruktur hydrodynamisch unterschiedlich exponierter Außen- und Innenriffe. - Helgoländer Meeresunters. 39, 375-417.

Mergner, H. \& Svoboda, A., 1977. Productivity and seasonal changes in selected reef areas in the Gulf of Aqaba (Red Sea). - Helgoländer wiss. Meeresunters. 30, 383-399.

Moll, H. 1983. Zonation and diversity of Scleractinia on reefs off S. W. Sulawesi, Indonesia. Thesis, Leiden, $106 \mathrm{pp}$.

Pielou, E. C., 1966. The measurement of diversity in different types of biological collections. - J. theor. Biol. 13, 131-144.

Rosen, B. R., 1971. The distribution of reef coral genera in the Indian Ocean. - Symp. zool. Soc. Lond. 28, 263-299.

Rosen, B. R., 1981. The tropical high diversity enigma - the corals' - eye view. In: Chance, change and challenge. Ed. by P. L. Forey. Cambridge Univ. Press, London, 103-129.

Scheer, G., 1971. Coral reefs and coral genera in the Red Sea and Indian Ocean. - Symp. zool. Soc. Lond. 28, 329-367.

Scheer, G., 1984. The distribution of reef-corals in the Indian Ocean with a historical review of its investigation. - Deep Sea Res. (A) 31, 885-900.

Scheer, G. \& Pillai, C. S. G., 1983. Report on the stony corals of the Red Sea, - Zoologica, Stuttgart $133,1-198$.

Schuhmacher, H., 1975. Die Rolle der Weichkorallen (Alcyonacea, Octocorallia) - innerhalb der Riffbiozönosen des Roten Meeres und des australischen Großen Barriereriffs. - Verh. dt. zool. Ges. 67, 380-384.

Stehli, F. G. \& Wells, J. W., 1971. Diversity and age patterns in hermatypic corals. - Syst. Zool. 20, $115-126$.

Svoboda, A, 1978. In situ monitoring of oxygen production and respiration in Cnidaria with and without zooxanthellae. In: Physiology and behaviour of marine organisms. Ed. by D. S. McLusky \& A. J. Berry. Pergamon Press, Oxford, 75-82.

Vine, P. J., 1974. Effects of algal grazing and aggressive behaviour of the fishes Pomacentrus lividus and Acanthurus sohal on coral-reef ecology. - Mar. Biol. 24, 131-136.

Vine, P. J. \& Vine, M. P., 1980. Ecology of Sudanese coral reefs with particular reference to reef morphology and distribution of fishes. - Proc. Symp. Coastal and Marine Environment of the Red Sea, Gulf of Aden and Tropical Western Indian Ocean, Khartoum 1, 88-140.

Webb, D. J., 1974. The statistics of relative abundance and diversity. - J. theor. Biol. 43, 277-291.

Weber, J. N. \& White, E. W., 1974. Activation energy for skeletal aragonite deposited by the hermatypic scleractinian coral Platygyra. - Mar, Biol. 26, 353-359.

Wells, J. W., 1954. Recent corals of the Marshall Islands. - Prof. Pap. U. S. Geol. Surv, 260-I, $385-486$.

Wijsman-Best, M., 1977. Indo-Pacific coral species belonging to the subfamily Montastreinae Vaughan \& Wells, 1943 (Scleractinia - Coelenterata) Part I. The genera Montastrea and Plesiastrea. - Zoöl. Meded. 52, 81-97.

Wijsman-Best, $M_{2}, 1980$. Indo-Pacific coral species belonging to the subfamily Montastreinae Vaughan \& Wells, 1943 (Scleractinia - Coelenterata) Part II. The genera Cyphastrea, Leptastrea, Echinopora and Diploastrea. - Zoöl. Meded. 55, 235-263.

Wilson, B. R. \& Marsh, L. M., 1980. Coral reef communities at the Houtman Abrolhos, Western Australia, in a zone of biogeographic overlap. - Proc. Int. Symp. Mar. Biogeogr. Southern Hemisphere, 259-278. 